\title{
Der Klassiker strebt zu neuen Ufern
}

\begin{abstract}
Sitagliptin hat den breitesten Zulassungsstatus unter den DPP-4-Hemmern. Aber der Hersteller hat noch mehr vor, wie beim ADA-Kongress in San Diego zu erfahren war. So möchte man die Datenbasis für den Einsatz bei älteren und niereninsuffizienten Typ-2-Diabetikern ausbauen. Zudem wird eine Zulassung der Fixkombination Sitagliptin plus Metformin als Initialtherapie angestrebt, ebenso wie die einer Fixkombination des Gliptins mit Simvastatin.
\end{abstract}

Mit der Einführung des ersten DPP-4-Hemmers für Typ-2-Diabetiker im Jahr 2007, Sitagliptin (Januvia ${ }^{\circ}$, übernahm das Unternehmen MSD eine Vorreiterrolle, es folgte die Fixkombination mit Metformin (Janumet ${ }^{\star}$ ). Inzwischen ist das Gliptin oral vielfach kombinierbar, mit Insulin und als Monotherapie zugelassen. Die meisten Erkenntnisse aus Zulassungsstudien basieren aber auf Daten von Patienten jünger als 65 Jahre. „Unser Interesse gilt derzeit daher besonders den älteren Typ-2-Diabetikern", sagte Dr. Barry J. Goldstein, Leiter des Therapiebereichs Diabetes/Endokrinologie bei den Merck Research Laboratories. Die Gruppe der Über-65-Jährigen profitieren ihm zufolge besonders vom guten Sicherheitsprofil des Gliptins, da sie oft multimorbide und verstärkt hypoglykämiegefährdet sind. Insgesamt wirke Sitagliptin bei alten Menschen so effektiv und sicher wie bei jüngeren. Es sei im Hinblick auf unerwünschte Effekte aber viel positiver als die oft von Älteren genutzten Sulfonylharnstoffe, so Goldstein.

Das bestätigte auch eine Post-hoc-Analyse zweier randomisierter Studien, die als Poster beim ADA vorgestellt wurde [1]. Darin war der Effekt von Sitagliptin im Vergleich zu Glipizid oder Glimepirid, jeweils zusätzlich zu Metformin, bei 460 Typ-2-Diabetikern $\geq 65$ Jahre untersucht worden. Es ergaben sich ähnliche $\mathrm{HbA}_{1 c}$-Senkungen wie mit der jeweiligen Vergleichskombination ( $-0,4$ bis $-0,7 \%$ - Pkte.), aber eine leichte Gewichtsabnahme und signifikant weniger Hypoglykämien mit dem Gliptin. Auch in einer im Mai publizierten 24-Wochen-Studie hatte das Gliptin in Monotherapie vs. Placebo seine gute Wirksamkeit und Verträglichkeit bei Patienten über 65 bis zu 96 Jahren (Mittel 72 Jahre) belegt [2].

\section{Bald auch bei schwerer Niereninsuffizienz?}

Geht es nach Goldstein, werden auch moderat bis schwer nierenkranke Typ-2-Diabetiker in Europa in absehbarer Zeit mit Sitagliptin versorgt werden können. Während der Wirkstoff in den USA von Beginn an in reduzierter Dosis bei moderater $(50 \mathrm{mg} / \mathrm{d}$ statt $100 \mathrm{mg} / \mathrm{d})$ und schwerer $(25 \mathrm{mg} / \mathrm{d})$ Niereninsuffizienz zugelassen war, und laut Goldstein dort auch viel genutzt wird, rät man hierzulande bisher davon ab. Dabei sei die Anwendung sicher, denn die Nierenfunktion werde vom Wirkstoff nicht beeinträchtigt. Die geringere Dosis sei auch nicht weniger wirksam, da die verlangsamte renale Elimination ausreichende Wirkspiegel ermöglicht. Für die nächsten sechs Monate stellte Goldstein weitere Daten dazu in Aussicht.

\section{Kombinierte Initialtherapie macht Sinn}

Von den beim ADA vorgestellten Studien hob Goldstein auch eine weitere Post-hoc-Analyse zur Fixkombination Sitagliptin plus Metformin hervor [3]. Darin war der Effekt der Kombination versus Metformin allein bei 365 bisher antidiabetikanaiven Typ-2-Diabetikern geprüft worden. Wie die DDG empfehlen die Leitlinien der US-Endokrinologen AACE/ACE ${ }^{*}$ ein $\mathrm{HbA}_{1 c}$-Ziel von max. 6,5\%. Nach 18 Wochen hatten fast $50 \%$ der initial kombiniert behandelnden Patienten dieses Ziel erreicht, aber nur 23\% derer mit der Monotherapie, ein signifikanter Unterschied. Der $\mathrm{HbA}_{1 \mathrm{c}}$-Abfall bei einem Ausgangswert von 7,5-9\% lag im Mittel bei 1,5\%-Punkten mit der Kombination und bei nur einem mit der Monotherapie. Goldstein: „Die meisten Patienten brauchen zum Erreichen des $\mathrm{HbA}_{1 c}$-Ziels eine Kombination, und damit früher zu beginnen als bisher ist sinnvoll." Darum arbeite man am Ausbau der Evidenzbasis für eine initiale Kombitherapie mit den beiden Wirkstoffen, die sich offenbar in ihrer Wirkweise gut ergänzen und im Effekt gar verstärken können [4].

\section{Gefäßschutz mit Statin in einer Tablette}

Die Brücke zwischen zwei Antidiabetika zu schlagen ist eine Sache. Neu wäre es, Gliptin mit Statin zu kombinieren und so den Gefäßsschutz bei Diabetes zu vereinfachen. Genau das ist laut Goldstein für den globalen Markt geplant. „In vielen Leitlinien wird für Diabetiker ein Statin empfohlen, aber oft nicht eingesetzt." Es wäre für die kardiovaskuläre Hochrisikogruppe der Diabetiker sicher eine Erleichterung, Glitpin und Statin in einer Tablette zu haben, sagte er. Von der Endpunktstudie TECOS ${ }^{* *}$ erhofft er sich zudem, die bisherigen Hinweise auf ein kardiovaskuläres Schutzpotenzial von Sitagliptin an einem Kollektiv von 14000 kardiovaskulär vorbelasteten Diabetikern erhärten zu können. Erste Daten der Studie werden voraussichtlich für das Jahr 2015 erwartet.

Sarah Louise Pampel

Quelle: Interview und Poster beim ADA-Kongress 2011 in San Diego

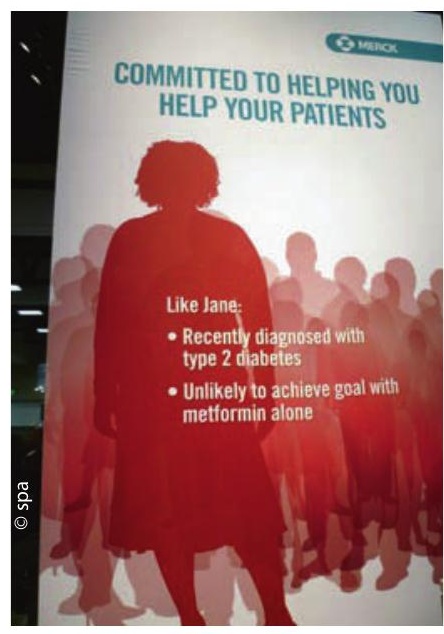

${ }^{*}$ American Association of Clinical Endocrinologists und American College of Endocrinologists ** Trial Evaluating Cardiovascular Outcomes With Sitagliptin

1. Diabetes 2011;60 (Suppl.1):A620 2. Current Medical Research \& Opinion 2011;27(5):1049-58

3. Diabetes 2011;60(Suppl.1):A290 4. Diabetes 2011;60(Suppl.1):A268 